\title{
The psychological impact of infertility. Directions for the development of interventions
}

\author{
Ana-Maria Vioreanu \\ University of Bucharest, Bucharest, Romania
}

\begin{abstract}
Introduction: Infertility represents a major health problem that oftentimes is experienced as a crisis situation. Because it interposes with achieving an important life goal, that of having children, its implications on the psycho-emotional level are robust.
\end{abstract}

Purpose: The present article aimed at exploring the multidimensional effects of infertility on mental health, as well as suitable psychological intervention strategies for patients both at an individual and couple level.

Methodology: An extensive literature review was conducted to select the most relevant information. Articles included were searched in PubMed, Google Scholar and other similar databases within a seven years interval (2014-2021) to assure the up-to-date quality of the paper. The main criteria of inclusion were: studies about the consequences of infertility on mental health, epidemiology, risk and protective factors, psychological models for infertility, psychological evaluation, established instruments, psycho-social and therapeutic interventions, intervention protocols used in public health for infertility patients.

Results and Discussion: Infertility is often experienced as a crisis situation, in which the most common consequences are visible at the level of mood disorders (e.g. anxiety, depression, marked distress) and at the social level (stigma, divorce, social isolation, financial difficulties). Psychological counseling is needed throughout medical treatment and therapeutic interventions (e.g. cognitive-behavioral therapy) have shown favorable results in managing the emotional balance of the individual/couple and reducing the risk of psychopathology.

Conclusions: Research highlights the beneficial effects of psychological interventions on reducing levels of stress related to infertility, anxiety and depressive symptoms and on improving the quality of life and couple dynamics.

\section{Keywords}

infertility, risk factors, protective factors, psychological interventions, infertility-related stress

Address for correspondence:

Ana-Maria Vioreanu. University of Bucharest. 90 Panduri Str., 050663, Bucharest, Romania

E-mail: ana.vioreanu8@gmail.com

This work is licensed under a Creative Commons AttributionNonCommercial 4.0 International License (CC BY-NC 4.0).

\section{(c) (i) (8)}

Submitted for publication: 10 August 2021

Revised: 07 October 2021 Accepted for publication: 24 October 2021

(C) Copyright: Vioreanu, 2021

Licensee NDSAN (MFC- Coordinator of the NDSAN), Italy doi: https://doi.org/10.32437/mhgcj.v4i1.113 


\section{Introduction}

According to the World Health Organization, infertility is the inability of a couple to obtain a pregnancy after twelve months of regular unprotected sex or after repeated attempts by the woman to become pregnant. Infertility is a major public health problem worldwide, affecting around 48 million couples and 186 million individuals of reproductive age. A distinction is made between primary infertility - the inability to have a pregnancy - and secondary infertility - the inability to have another pregnancy after a previous successful one. The impact of infertility can have major consequences both psychologically and socio-economically. The first to suffer from these are women, as they are prone to stigma, an increased risk of domestic violence, divorce, acute distress, anxiety, depression and social isolation (WHO, 2020).

One of the strongest predictors of infertility is the advanced age of the woman, but an equally important role is played by environmental factors and lifestyle. Factors influencing reproductive capacity may be gender specific, but this is not a condition (Hart, 2016).

There are several terminologies explaining this issue, so it is necessary to distinguish between them. First, infertility "is defined as a disease characterized by failure to establish a clinical pregnancy after 12 months of regular and unprotected sexual intercourse due to a person's impairment of reproductive capacity either individually or as a couple." (Borght \& Wyns, 2018, p. 2). Infertility is a disease that causes disability as an impairment of the function.

Subfertility is also the effort of couples to achieve a pregnancy, associated with any low degree of fertility. According to the International Classification of Functioning, Disability and Health (ICF), it is coded b660, affecting procreation functions (functions associated with fertility, pregnancy, birth and lactation) - b6600 fertilityrelated functions with impairments such as subfertility and sterility (World Health Organization, 2001). The terms infertility/ subfertility are used interchangeably.

Second, while infertility is a limited condition over a period of time, sterility indicates a permanent stage of infertility (Zegers-Hochschild, et al., 2017).

Regarding the prevalence of infertility, it is estimated to affect one in seven couples of reproductive age in Western countries and one in four couples in developing countries. There are regions around the world that are more prone to infertility such as South Asia, the Middle East, North Africa, Eastern and Central Europe, where the index can reach $30 \%$. The contribution of female and male factors differs: men are involved in 50\% of cases, with differences between certain regions of the world. Globally, infertility affects between 8 and $12 \%$ of couples, with secondary infertility being the most common. One explanation for this is the risky methods of abortion, the lack of adequate care in maternity wards, which can lead to dangerous infections after birth or abortion (Borght \& Wyns, 2018).

Female factors that contribute to infertility may be related to other biological disorders. The International Statistical Classification of Diseases and Related Health Problems (ICD-10-AM) classifies infertility as follows: N97 female infertility (inability to complete a pregnancy) with the following etiologies:

- N97.0 Female infertility associated with anovulation;

- N97.1 Female infertility of tubal origin;

o Associated with congenital anomaly of the fallopian tube:

- locked;

- occlusion;

- stenosis;

- N97.2 Female infertility of uterine origin;

- Associated with congenital uterine abnormality;

o Non-implantation of ovum;

- N97.3 Female infertility of cervical origin;

- N97.4 Female infertility associated with male factors:

- N97.8 Female infertility of other origin;

- N97.9 Female infertility, unspecified (World Health Organization, 2002).

Going further and analyzing demographics around the world, there are six important facts about demography:

1) Millions of people worldwide suffer from this condition;

2) The highest infertility rates are those of secondary infertility and are found mainly in contexts poor in resources;

3) Africa is the region with the most alarming infertility rates globally;

4) Africa suffers from a demographic paradox: high infertility rates coexist with high fertility rates;

5) Although this issue is a major global crisis, there is an acute lack of prevention programs and treatment services - the main justification being directly linked to a form of control of overpopularization, especially in regions with a very high birth rate (e.g. sub-Saharan Africa);

6) The regions that suffer the most from infertility are the same that offer the fewest options of diagnosis and access to treatment (Inhorn \& Patrizio, 2015). 


\section{Purpose}

The main objective of this paper was to explore and analyze the psychological impact of the infertility condition, with all its multidimensional effects. More and more recent research draws attention to the risk factors for infertility and to the widespread effects of this condition at the biopsycho-social level. That is why psychological intervention in managing this issue is urgently needed, and the literature suggests scientifically validated methods that can restore psychological balance. Starting from psycho-education and understanding the medical condition, to evaluation and psychological intervention, each step must be followed in order to bring the patient back to the psychological safety zone, to help him face the challenges that follow and to support him in taking those better informed decisions. Therefore, psychological support is essential from the moment of diagnosis until the end of treatment, regardless of its results.

\section{Methodology}

The present study is a literature review intended to serve as a practical guide for mental health specialists for the development of individualised interventions for infertility patients. Articles included were searched in PubMed, Google Scholar and other similar databases within a seven years interval (2014-2021) to assure the up-to-date quality of the paper using keywords such as: "Infertility-related stress", "Risk factors", "Psychological impact", "Counseling", "Interventions". The main criteria of inclusion were: studies about the consequences of infertility on mental health, epidemiology, risk and protective factors, psychological models for infertility, psychological evaluation, established instruments, psycho-social and therapeutic interventions, intervention protocols used in public health for infertility patients. The exclusion criteria were the year of publication (older than 2014) and the source of publication (low impact factor).

\section{Results}

\section{Risk factors and consequences of infertility}

According to the literature, there are many factors that can influence the spontaneous fertility of the couple. These include:

- Reproductive system disorders: congenital disorders, muscle tissue gland, endometriosis, polyps and anatomical problems;

- Genital infections or sexually transmitted diseases;

- Old age: the quality of fertility begins to deteriorate at the age of 25-30 years in women and studies show that most of them are not aware that postponing a pregnancy increases the risk of infertility. Moreover, it is erroneously considered that medical treatments used to have and maintain a pregnancy can solve the problem of declining fertility caused by old age. The prognosis is all the more unfavorable as the woman's age is over 35 years, and the duration of infertility exceeds 3 years (Eijkemans, 2014);

- Hormonal dysfunctions (e.g. hypothyroidism, hyperprolactinemia);

- Smoking and alcohol consumption: studies show that $40 \%$ of men suffering from infertility are smokers. The impact of smoking is also great in women, as nicotine works by destroying eggs faster. A lost egg cannot be recovered, which is why women who smoke reach menopause up to 4 years earlier than normal age. Alcohol consumption in men indicates a reduction in sperm count (Sabarre, Khan, Whitten, Remes, \& Phillips, 2013);

- Obesity and nutrition: because the most common complication of obesity in women is polycystic ovary syndrome (between 30-70\% of women with this syndrome are obese) the risk of infertility is exacerbated (Tsai, et al., 2013).

- Sexual violence: research indicates that women who have a history of psychological trauma caused by sexual abuse have a three times higher risk of infertility (Deyhoul, Mohamaddoost, \& Hosseini, 2017).

- Testicular dysfunction and sperm quality;

- Negative life experiences and stress: studies in mice show how systematic stress affects reproductive function by inhibiting a key hormone in the reproductive system. Moreover, life history theory provides a frame of reference that explains the impact of adverse early life experiences on fertility from an evolutionary perspective. Specifically, people who experience unfavorable childhood conditions (e.g. lack of resources, family imbalances) are more likely to approach a fast life history strategy characterized by impulsive

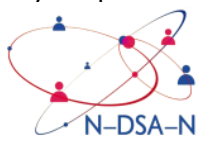


behaviors, early reproduction, prolonged physiological activation, and poor mental health management. All this translates into a major risk of infertility in adulthood (Schweiger, Schweiger, \& Schweiger, 2018).

Infertility itself does not show a certain somatic symptomatology, except when it is associated with other clinical conditions. Instead, perhaps the most common symptomatology of infertility is at a psychological level, as mental health can be affected in a proportion of 30 to $80 \%$ depending on the cause and duration of infertility (Luk \& Loke, 2015). The main symptoms can range from stress and an inferiority complex, to anxiety and major depression (Yusuf, 2016). Stress levels are much higher among women, for various reasons. Perhaps one of the most important reasons is the status of being a mother, a fundamental role in the life of a woman, which for many of them is considered defining and therefore an essential purpose in life. The inability to achieve this goal comes with many emotional imbalances, and can be a crisis situation that threatens the psychological well-being of women. In addition, some studies have suggested that among women suffering from infertility, the initial level of stress caused by the condition is compounded by additional stress, at least as strong, caused by medical treatments. The level of stress caused by infertility treatment is the second highest intensity of a stressor, after the death of a family member or divorce (Wiweko, Anggraheni, Elvira, \& Lubis, 2017).

The consequences of infertility extend to the family, social and economic level, the damage given by this condition being multi-dimensional. Next, some of the dimensions of this issue will be analyzed.

a) The psychological dimension: as mentioned above, perhaps the most affected area of life is the psychological one. The most common reactions observed among individuals and couples when they hear the news are the shock reaction, denial (which if prolonged can prevent access to early intervention), anger (perception of an injustice), the feeling of losing control (weakening coping mechanisms), social isolation, guilt (which can lead to depression), anxiety (Kırca \& Pasinlioğlu 2013; Sezgin \& Hocaoğlu 2014).

b) The socio-cultural dimension: culture, traditions and beliefs play a very important role in certain societies, putting their mark on issues related to the subjectivity of each person's health. For example, in developing societies, infertility can be a real social problem, with couples facing stigma and experiences that lead to shame and marginalization. These things happen because of the emphasis on various values, especially those related to family life, namely: social pressure to have children as soon as possible after marriage, portrayal of the child in the form of a supreme achievement that brings pride and prestige, the attribution of the parent to transfer certain norms and social roles further. For such cultures, the inability to fulfill their "mission" and social and family expectations can be categorized as a defect, a major disadvantage or can be understood as a bad will to have a child. Therefore, if they are unable to achieve their social roles defined by their own culture (women, the role of mother and men, the role of male example), people suffering from infertility are at risk of developing feelings of lack of self-worth, decreased self-esteem and the perception that there is something wrong with them as a family unit (Patel et al., 2018; Pinar \& Yildirim, 2016).

The economical dimension: infertility treatments are expensive and long-lasting, which is why couples who are part of resource-poor areas may be unable to gain access to treatment. Moreover, in some cultures (mainly in developing countries) the inability to give birth to a child comes with a breakdown of a woman's economic security, caused by various consequences: divorce, alienation from family members, withholding of any financial resources provided by the family until then and even confiscation of the inheritance (Rouchou, 2013). 


\begin{tabular}{lll}
\hline \multicolumn{1}{c}{ Medical treatment } & involves all fertility treatments aimed at \\
\hline Assisted Reproduction Technology (ART) & $\begin{array}{l}\text { manipulating either the egg or the } \\
\text { embryo }\end{array}$ \\
in vitro fertilization (IVF) being the \\
most widely used procedure worldwide
\end{tabular}

\begin{tabular}{l}
\hline Perfusion of sperm from the fallopian tube \\
\hline Agents that induce ovulation \\
\hline Intra-fallopian gamete transfer
\end{tabular}

\section{Fig 1. Treatment options for infertility}

Primary prophylaxis is the most useful method of reducing the frequency of infertility, but most of the time it is not applied. In order for the prevention to be effective, access to information about the disease and risk factors is needed. Research has indicated that, including among women with a high level of education, the significant impact of advanced age on infertility or the risks associated with reproductive assistance technology remain unknown (Hammerberg, et al., 2017). Since a crucial step in preventing infertility is to implement change into lifestyle and certain deleterious habits, being informed about what to do in order to achieve a healthy pregnancy is the basis for active strategies of help seeking and preventing the secondary effects of a possible diagnosis (e.g. distress, anxiety).

Unfortunately, most people only start to inform about this when they are directly confronted with the problem, and worries and feelings of fear have already set in, putting pressure on the effectiveness of treatments. Therefore, the role of prevention is to meet the educational needs of people who want children, to protect them from the psychological shock of a potential diagnosis of infertility, but also to promote good health practices overall. The promotion of psychoeducational programs on this topic, but also free access to scientific information, are two key points of primary prophylaxis (Öztürk, Siyez, Esen, \& Kağnici, 2020).

\section{Psychological aspects involved in infertility issues}

The psychology of infertility began to take shape in 1930, with the introduction of the psychogenic model of infertility by Berg and Wilson, in which it was postulated that a major cause of infertility unexplained medically is represented by psychopathology. More precisely, the etiological factor of unexplained infertility would be a psychic conflict of different nature: conflicting sexual identity, conflicting relationship between mother and child (Berg \& Wilson, 1991).

Over time and with the advancement of research and technology in gynecology, the psychogenic model has lost its validity, proving that the probability that unexplained infertility is caused exclusively by psychological problems is extremely low and that, in most cases, it is basically a biomedical problem (e.g. pelvic pathology). However, the contribution of the psychogenic model has been considerable, setting the starting point for psychological research in the field of obstetrics and gynecology (Boivin \& Gameiro, 2015).

A major influence in the psychological study of infertility was launched by the advancement of multifactorial models, one of the best known being the bio-psycho-social model. It attests to the contribution of various factors (biological, psychological, social, environmental) in the etiology of somatic diseases. Thus, there may be several determining factors and consequences of diseases, which manifest themselves differently from person to person, depending on the personal history, individual differences, various developmental environments, etc. (Lipowski, 1984).

One current direction of research that continues to receive much attention is the link between stress and infertility. This field is developed through a solid body of studies that focuses on many aspects starting from individual characteristics (such as gender, type of stressor, life events), explanatory mechanisms (lifestyle, hormones, compliance with treatment) and reaching up to protection factors and intervention models (such as counseling, psycho-education, help-seeking behavior). Another aspect that 
deserves a glimpse of change it is the excessive focus on female infertility, losing sight of the male factors that can contribute to this issue. Especially in the psychiatric debate of infertility, men tend to be included much less often in research and therefore neglected during treatment. That is why recent studies recommend shifting attention to the area of dyadic models of conceptualization and intervention (Boivin \& Gameiro, 2015).

Continuing along the lines of psychological models of infertility, we review a theoretical framework that discusses several variables that can lead to the development of specific intervention models. The Interaction Model of Client Health Behavior (IMCHB) argues that the interaction between intrapersonal and contextual factors is what contributes to the client's healthy behavior. Shortly, clients present two important sets of variables, namely, background variables demographics, social influences, medical history, environmental resources - and dynamic variables - intrinsic motivation, affective response, cognitive appraisal - where the firsts are relatively stable over time, and the dynamic ones are influenced by environmental factors. Therefore, dynamic variables are intrapersonal, and due to their modifiable nature, are targeted in behavioral interventions designed to support health-oriented behavior (Cox, 1982). This model has a major impact in promoting behavioral tactics designed to reduce the risk of poor health (for example, by changing lifestyle and trying to eliminate vicious behaviors).

Most often, infertility takes the form of a crisis situation, putting in danger the psychological wellbeing of the individual/couple, life satisfaction and other aspects of mental health. It is useful to conceptualize the crisis so that we understand how to deal with the problem in the most effective way (Fig. 2).

Summing up the above, we can make a general observation on the areas of research in infertility. Two main directions are those of medicine and clinical psychology and those of social models. From this point of view, infertility research is a vast, eclectic field, focusing on the essential aspects of each field. For example, the clinical perspective turns its attention to psychological distress as a direct result of infertility, coping mechanisms and effective methods to identify those who need prompt intervention. On the other hand, the social perspective draws attention to several concepts such as healthoriented behavior, seeking help, the social role of being a parent, stigma, marital dynamics, gender differences, culture, and individual/dyadic differences (Johnson, Greil , Shreffler, \& McQuillan, 2018).

Anxiety and depression are the most common psychopathologies in infertile couples for several reasons: uncertainty about the cause of infertility and the success rate of treatment, uncertain duration of treatment, financial difficulties and social pressure. Of all these, the strongest predictor of depression is the duration of treatment. On the one hand, because a long duration of treatment can lead to trial failures and a possible chronicity of the disease. On the other hand, the longer the duration of treatment, the greater the risk that the identity of the disease will be known to more friends and family members, which can lead to social pressure, stigma or feelings of shame (Maroufizadeh, et al., 2018). A current review of the literature indicates that the symptoms of clinical intensity manifested occur in 25 to $60 \%$ of people affected by infertility (De Berardis, et al., 2014). Moreover, research draws attention to the fact that high levels of depression in women are associated with avoiding medical treatment and increasing dropout rates after the first cycle of treatment. Consequently, the reasons for giving up treatment or refusing specialized help are explained by psychological factors (Rich \& Domar, 2016).

Unfortunately, the complications of depressive symptoms and acute stress have effects on the success rate of treatment, forming a kind of vicious circle. In short, stress produces harmful effects on the hormonal system, with implications on sexual functions and the body's response to treatment (Ergin, et al., 2018). Therefore, psychological interventions concomitant with medical treatment specific to infertility are a necessary condition in restoring the equilibrium of the individual/couple and in removing obstacles to the success of treatment. 


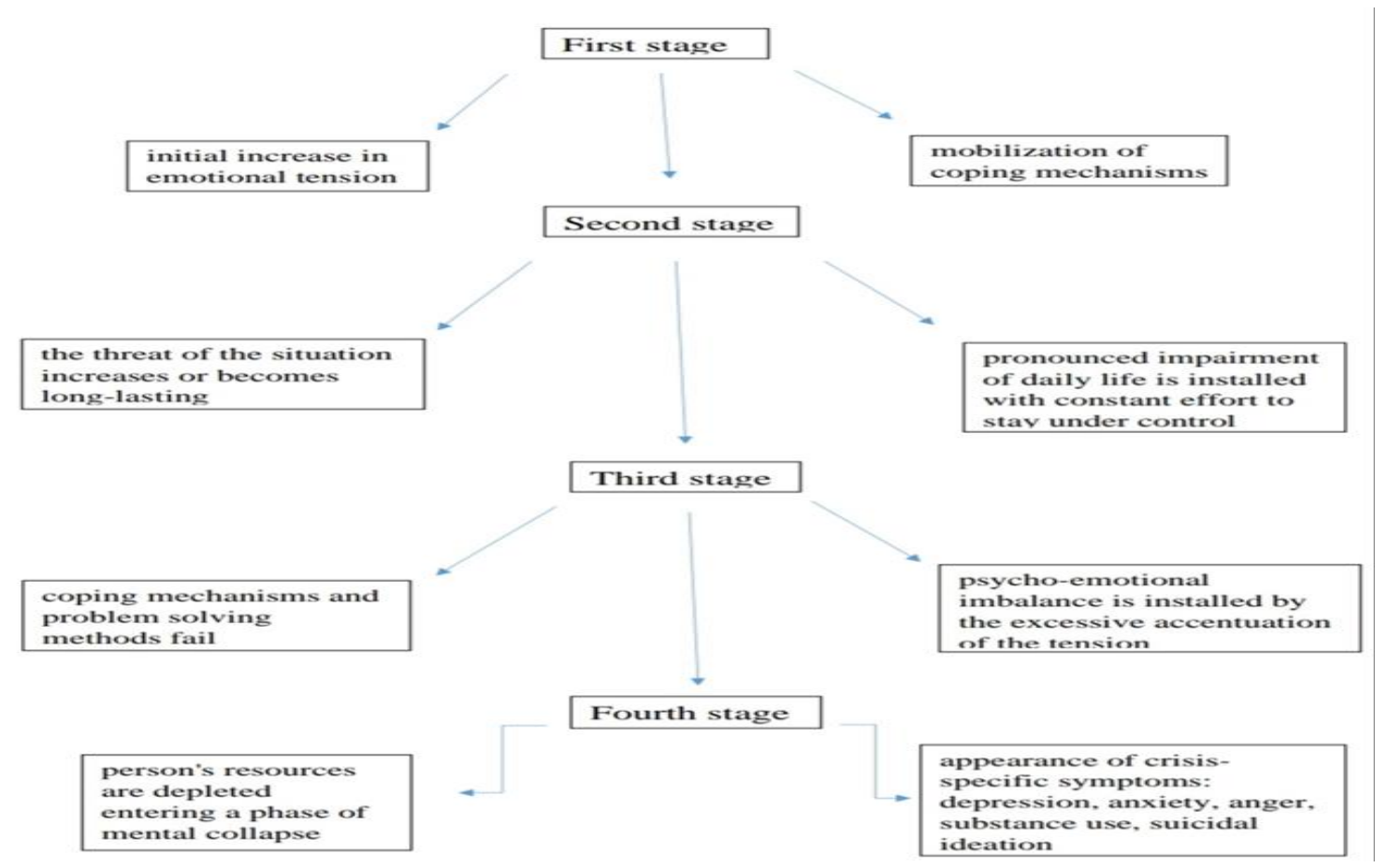

Fig.2 The four-step model of crisis response (Caplan, 1964).

\section{Psychological evaluation in infertility}

When it comes to estimating the impact of a diagnosis of infertility on the functionality and psycho-emotional balance, it is important to analyze the aspect of psychological evaluation. Psychological evaluation should be done both in the initial stage, immediately after the diagnosis and before the start of medical treatment, and after the implementation of the treatment plan and throughout it.

When the patient is in the initial stage, that of learning about the diagnosis, one of the therapeutic goals should focus on exploring his concerns and fears about what is to come and on psychological preparation, strengthening internal resources for treatment requirements. An important first step in this process is the correct information of the patient about the condition he suffers from, the treatment possibilities and the expected results. Thus, the patient will know the details necessary to form realistic expectations and will lay the foundations for informed decision making.

The literature emphasizes a person-centered approach to counseling, and the U.S. Institute of Medicine has even provided a definition of what it means to provide patient-centered health care: "Providing care that respects and responds to the patient's individual preferences, needs and values and ensuring that patient values guide all clinical decisions" (Institute of Medicine, 2001). Taking into account the specific needs of the patient, the risk factors for high distress can be analyzed, depending on his history and individual differences: pre-existing psychological disorders, attitudes towards parental status, coping mechanisms, quality of couple relationship, social support network and treatment-related factors such as side effects, success rate and others (Doyle \& Carballedo, 2014).

The main objective of the psychological evaluation of the patient with infertility will capture the emotional status and reactions/attitudes towards infertility before starting treatment. For the evaluation to be as valid as possible, it needs to be structured on several levels:

a) Gathering information

- from the personal, medical, professional, psychiatric history, both of the individual and of couple;

- Establishing the current level of functionality;

- Checking the impact of infertility on mental health, marital stability, reproductive and sexual history;

- Administration of psychological tests.

b) Clinical interview

- Necessary for completing the clinical picture, favoring the evaluation and construction of an intervention plan; 
- Combining psychological testing with the clinical interview provides an appropriate assessment and identification of the patient's psychopathology and immediate needs.

Therefore, a correct psychological assessment will include theoretical, clinical and therapeutic principles, so as to ensure the quality of services provided, the implementation of effective treatment and / or the application of counseling sessions to support the patient / couple in the medical process (Covington \& Burns, 2006).

When it comes to psychometric assessment, the main indicators to consider are stress / distress, coping strategies, social support, quality of life, adaptation to treatment, helplessness, acceptance, anxiety and depression. An important aspect here refers to the use of instruments to measure the specific valences of infertility, as general psychological scales cannot provide sensitivity and specificity for infertility. Thus, more than ten instruments have been developed to measure the impact of infertility at the psychological level and to capture vulnerable areas or protective factors (Pedro, et al., 2016). These include: Fertility Problem Inventory (FPI; Newton et al., 1999), Fertility Quality of Life Tool (FertiQoL; Boivin et al., 2011), COMPI - Fertility Problem Stress Scales (COMPI-FPSS; Schmidt, 2006), SCREENIVF (Verhaak et al., 2010), FertiSTAT (Bunting et al., 2010).

Fertility Problem Inventory (FPI) is one of the first multidimensional questionnaires built to capture the most relevant aspects to consider in the therapeutic process in patients with infertility. It is a self-report questionnaire and can be completed by both members of the couple. The tool shows considerable gender differences in the effect of stress on perceived anxiety and depression symptoms, self-reported resilience levels, and marital satisfaction, both from a female and male perspective (Zurlo, Cattaneo Della Volta, \& Vallone, 2017).

Fertility Quality of Life Instrument (FertiQoL) is a tool that focuses primarily on measuring the quality of life of patients with infertility and is a standard in the psychological assessment of infertility. It consists of two parts, namely: FertiQoL Central and an optional treatment module that measures quality of life levels throughout medical treatment.

FertiQoL Central consists of 4 scales totaling 24 items that measure the emotional, mind-body, relational and social dimensions. The treatment module contains 10 items plus 2 more general items that measure the well-being of physical health and of life in total. FertiQoL also presents the necessary psychometric qualities, being validated in several countries (see www.fertigol.org) (Aarts et al., 2011; Donarelli et al., 2016).
COMPI (Copenhagen Multi-Center Psychosocial Infertility) - Fertility Problem Stress Scales (FPSS) is a key tool used in assessing infertility related stress on a global level. It was built on the basis of the Fertility Problem Stress Inventory (Abbey et al., 1991) and patient interviews (Schmidt, 2010) in order to determine risk factors for infertility distress that may interfere with treatment, but also protective factors and gender differences (Sobral, Costa, Schmidt, \& Martins, 2017).

The other two mentioned instruments, SCREENIVF and FertiSTAT, are two very useful screening tools in the rapid evaluation of the patient immediately after diagnosis. SCREENIVF (Ockhuijsen et al., 2017) indicates the patient's risk of developing a psychopathology at the end of infertility treatment, and FertiSTAT (Blanchet et al., 2019) provides support in assessing knowledge about the pathology and helps increase awareness of associated risk factors for infertility. The latter consists of a list of indicators representing the main risk factors for low fertility in women and men, where each indicator is coded by a color signifying the severity of the factor (blue, yellow, orange and red - for more details see http://www. fertistat.com/).

\section{Discussion}

\section{Psychological intervention strategies for patients with infertility - Opportunities and Challenges}

The role of psychological counseling in infertility highlights essential issues both individually and as a couple or group. Counseling should be a step that every patient with infertility goes through, as the implications at the psychological level, but also at the level of the treatment plan, are essential.

In the initial stage of psychological counseling, the patient benefits from a framework based on unconditional acceptance, empathy and active listening, so as to be motivated to seek and find the most appropriate solutions for him. The European Society of Human Reproduction and Embryology (ESHRE, 2002) explains how the process of psychological counseling gives the patient the opportunity of making informed decisions when it comes to medical treatment for infertility. Basically, the counselor prepares the person to start treatment, provides support throughout the treatment and helps them adapt to the different outcomes that treatment procedures may have. Moreover, the counselor facilitates the consideration and adoption of alternatives in case of treatment failure (e.g., 
adopting a child or accepting a happy child-free life) (Joy \& McCrystal, 2015).

There is a growing trend in research to identify the most effective types or strategies of psychosocial intervention. This imposes a challenge for the mental health practitioner as research in recent years has failed to establish a clear methodology due to mixed results. For example, some meta-analyzes (Ying et al., 2016; Chu et al., 2017) have suggested that psychological interventions lead to a significant reduction in negative affect caused by infertility, help increase the likelihood of becoming pregnant, and improve marital function ( Frederiksen et al., 2015). On the other hand, there have been meta-analyzes that have indicated the lack of significant effects of psychological interventions on the chance of becoming pregnant and on the decrease in stress levels (Boivin, 2003). Therefore, it is imperative to investigate the effectiveness of psychological interventions in managing the stress caused by infertility and their possible effects on the pregnancy rate and satisfaction in the couple. Next, it is important to describe the psychological interventions that the literature indicates to be the most useful to apply to patients with infertility.

One of the interventions that has enjoyed significant positive results in reducing distress caused by infertility is cognitive-behavioral therapy (CBT). Numerous studies have suggested that CBT techniques are useful in managing perceived stress and infertility-related mood disorders (such as anxiety) (Zhou, Cao, Liu, \& Xiao, 2021). Such techniques include relaxation methods (autogenic training, progressive relaxation), stress inoculation training (SIT), cognitive restructuring (techniques for identifying and modifying distorted cognitions induced by automatic thoughts about infertility; e.g. catastrophization, global evaluation), problem-solving and decision-making techniques, and lifestyle-related education (nutrition, exercise, sleep and work schedule) (David, 2017). Some studies included in this metaanalysis (Greil et al., 2014) and which used CBT as a psychological intervention, showed that patients report significant improvements in mood, stress management and marital satisfaction at 6 months post-intervention. Moreover, progress tends to be sustained over time, with participants indicating lower scores for depression 1 year after treatment.

In order to create a clearer picture of how CBT sessions are conducted, we can take as an example the randomized control study of Karaca et al. (2019), in which a classic CBT intervention protocol was followed. In this study, the experimental group benefited from a group cognitive-behavioral therapy program over 11 weeks. The sessions lasted on average, two hours, only the first and last one having 20 extra minutes to complete the pre and post intervention questionnaires. The first session focused on the patient's psycho-education both on the disease (with the help of an obstetrician) and on drug and psychological treatment options. The next two sessions focused on clinical assessment (assessment of the patient's condition and needs) and conceptualization of the case (a comprehensive explanation of the patient's clinical picture). The next six sessions were key to therapy, implementing cognitive-behavioral stress management methods and techniques such as progressive muscle relaxation, breathing control techniques, planning and goal setting, methods for identifying and disputing negative thoughts and ways to replace them with adaptive ones that contribute to psychological well-being. Furthermore, the participants were supported in exploring the maladaptive coping mechanisms they used until then to cope with the symptoms of anxiety and depression, in order to improve them and to become more effectively. Participants were given homework after each session, as they help to strengthen the skills learned in therapy. Session ten aimed to address other issues specific to infertility, namely the couple's sexual problems. In the last session, a general conclusion was made based on the summary of the key points of each meeting and indications were given for strategies that can be adopted to prevent relapses.

The results of this study suggest that group cognitive-behavioral therapy has significant positive effects on the control group, on infertility related stress, symptoms of anxiety and depression and on general health. These results are conclusive with other studies, which point to the high efficacy of CBT (individual) in reducing global stress caused by infertility, with post-test results indicating a considerable decrease in stress and anxiety / depressive symptoms compared to the initial results (Faramarzi et al., 2013; Springer et al., 2018).

Another therapeutic intervention very often used in the areas of health psychology is based on the principles of mindfulness meditation. The fundamental principle of mindfulness meditation is the awareness of the present moment, sustained attention, giving up judging what is happening here and now (negative thoughts / emotions) and practicing gratitude (Bai, et al., 2019). Evidence from the literature shows the effectiveness of mindfulness-based interventions in reducing anxiety and depressive symptoms (Eisendrath, et al., 2016) reducing chronic pain (Morone, et al., 2016) and increasing quality of life (Montgomery et al., 2016). The ability to be flexible and shift your

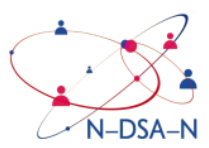


attention to what you are experiencing right now helps to integrate and accept thoughts and emotions, ultimately leading to detachment from their negative valences (e.g., in case of depression, feelings lack of self-worth and lack of hope) (Creswel, 2017).

Therefore, interventions such as those based on mindfulness are useful and should be applied in reproductive medicine, as in addition to the longterm benefits specific to the patient, they may have other benefits such as limited costs and conservation of limited resources for infertility treatment.

A new therapeutic technique derived from the CBT area, belonging to the third wave of cognitivebehavioral therapies, is Acceptance and Commitment Therapy (ACT), which shows promising results in recent studies. This technique is based on certain essential processes such as acceptance, mindfulness (contact with the present moment), cognitive diffusion and actions accompanied by commitment, which aim to achieve and develop psychological flexibility (Hosseinpanahi, et al., 2020). Through this therapy, the patient learns to accept subjective experiences with all his thoughts, feelings, and perceptions, to limit excessive attention to himself as a victim, and, perhaps most importantly, the person learns how to identify his own qualities and internal resources and how to translate them into behavioral goals, to use them for easy adaptation to life experiences (Samadi \& Doustkam, 2014). ACT has been shown to be effective in interventions targeting mental disorders, but also chronic medical conditions (e.g. chronic pain). The main effects act on reducing psychological pain (suffering) and improving psychological wellbeing (Gloster, Walder, Levin, Twohig, \& Karekla, 2020).

To sum up, the literature captures three of the most useful therapeutic strategies that can be used in patients with infertility: cognitive-behavioral therapy, therapy based on mindfulness and therapy through acceptance and commitment. It comes as a challenge for the therapist to find and use the therapeutic strategy that best fits the client, as every person has its individual differences.

Furthermore, it is important to follow the instructions in the established practice guidelines to ensure the quality and efficiency of psychosocial services provided to patients with infertility. Such an example of guidelines is the guide by Boivin and collaborators (2001), which is divided into six sections that describe the objectives, purpose, communication skills required and potential problems that may arise in the psychosocial counseling of patients with infertility.

Briefly, the first section contains the necessary information on the medical consultations to be performed and how they differ from other types of consultations in the field of obstetrics and gynecology. The second section introduces the role of counseling the patient with infertility and highlights the most common challenges, who is more likely to need counseling, who can do counseling and what it entails. Section three, recalls patient-centered good practices and indicates ways to incorporate counseling into the medical treatment scheme. Section four focuses on factors that may determine the urgent need for counseling such as treatment failure, worsening psychological distress, or sexual dysfunction. Section five considers different ways of reproduction and discusses the ethical and moral issues they may impose (e.g. donating gametes, choosing a surrogate mother, or adoption for both heterosexual and homosexual couples). Section six mentions additional ways of psycho-social help that can be integrated with counseling in fertility clinics such as the delivery of psycho-educational materials, online / telephone counseling, help groups, self-help strategies. To illustrate as clearly as possible the content of this practice guide, the following examples can be provided:

- For example, in the case of psychoeducational materials, they must be written in a comprehensive way and in such a way that the patient does not feel excluded if he cannot identify with the situations described as an example. At the same time, it is important that the information provided is empirically supported, to capture the essential aspects of the issue (risk / protective factors, coping / resilience mechanisms) and to normalize the psychological consequences.

- In the case of support groups, social support is facilitated, options are offered to strengthen a meaningful life (child-free or by resorting to alternative methods of forming a family), strengthening coping strategies so that patients face the inter and intra-personal problems they may encounter.

- In the case of patients with high levels of psychological distress, clinical evaluation is performed and methods of therapeutic intervention are established in order to reduce the level of risk to psychopathology.

- In the case of patients who are at the end of medical treatment, the perceived effects of losing an important goal in the couple's life are addressed in therapy or counseling and the present condition of the partners is analyzed to 
observe the level of preparation before the end of treatment.

These are some of the essential examples for counseling the infertility patient exposed in this guide (Boivin, et al., 2001).

National Institute for Health and Care Excellence (NICE) in the United Kingdom is an international benchmark for good practice based on scientific evidence. The recommendations regarding the care of the patient with fertility problems emphasize the central role of the effects at the psychological level and how the information should be provided so as to be helpful to the patient. It mentions the need to inform both partners about the effects of stress caused by fertility problems, how it can affect sexual function, leading to reduced sexual contact and, therefore, to the spread of fertility problems. Furthermore, people suffering from fertility problems are encouraged to consult a mental health specialist, as addressing problems, investigations and medical treatments can exacerbate psychological stress, making the person vulnerable. Psychological counseling should be provided to the patient both at the time of diagnosis (pre-treatment), during and at the end of treatment (post-treatment), regardless of the results obtained. A final important principle for infertility counseling is that the person providing counseling should not be directly involved in the process of treating the clinical condition (NICE, 2017).

\section{Limitations of the study}

One of the limitations in the counseling of infertility patients is the lack of extensive prevention programs at a public level. Such programs are mainly based on primary prevention (prevention of the onset of the disease) and secondary (therapeutic actions taken for early detection and for slowing down the progression of the disease). An effective prevention strategy should consider eliminating, giving up, and reducing exposure to risk factors (e.g. smoking, unhealthy diet, sedentary lifestyle). Some of the clinical practices that have proven to be effective in prevention are medical checks to ensure the proper functioning of the reproductive system, screening for sexually transmitted diseases, prevention of addictive behaviors (smoking, substance use) in adolescents, smoking cessation in adults, promoting a healthy lifestyle through a balanced diet and constant sport (Borght \& Wyns, 2018).

Another limitation would be that this paper considers three widely-used and proven methods of therapeutic interventions for infertility patients. Nevertheless, there are other types of interventions that can help in the therapeutic process but were not mentioned here.

\section{Conclusions (and Future Work)}

The role of the mental health specialist is undeniable in the treatment process of infertility and research is increasingly pointing out its importance. For example, as mentioned in this paper, the patient-centered approach is the medical care that should be implemented in any clinic. If up until now, the role of the counselor was more in crisis management and providing information needed to make decisions, now his contribution is focused on the area of psychotherapy. Taking into account the most important responsibilities of the mental health specialist in the field of reproductive medicine, the following can be summarized:

- The mental health specialist has the task of creating and effectively implementing prevention / intervention programs specific to the patient's emotional needs, respecting the patient-centered approach, in order to increase awareness of the need for psychological support in such cases;

- Helps patients in decision making;

- Supports the development of adaptive coping mechanisms and helps to identify maladaptive patterns;

- Helps the patient prepare for the start and maintenance of treatment cycles (by developing psychological flexibility, resilience and other internal resources);

- Develops and implements psycho-social selfhelp tools based on empirical evidence for the patient to benefit from outside the clinic;

- Provides training to medical staff in order to develop communication skills with the patient and knowledge of the psychological implications of such a diagnosis;

- Develops programs and support groups for patients in the high risk area of psychopathology (Patel, Sharma, \& Kumar, 2018).

In conclusion, research clearly highlights the beneficial effects of psychological interventions on reducing levels of stress related to infertility, anxiety and depressive symptoms and on improving the quality of life and couple dynamics. It is important to have access to and promote such types of interventions, as many patients are reluctant to resort to them, although their implications have been proven and continue to be. Therefore, the practical objective of this paper is to provide an overview of the psychological impact of infertility and possible directions for 
designing of psychological prevention and intervention programs in infertility.

\section{Conflict of interest}

The author declares that she has no conflict of interests.

\section{References}

Aarts, J., van Empel, I., Boivin, J., Nelen, W., Kremer, J., \& Verhaak, C. (2011). Relationship between quality of life and distress in infertility: a validation study of the Dutch FertiQoL Human Reproduction, 26(5), 1112-1118. doi: https://doi.org/10.1093/humrep/der051

Aarts, J., van Empel, I., Boivin, J., Nelen, W., Kremer, J., \& Verhaak, C. (2011). Relationship between quality of life and distress in infertility: a validation study of the Dutch FertiQoL . Human Reproduction, 26(5), 1112-1118. doi: https://doi.org/10.1093/humrep/der051

Abbey, A., Andrews, F., \& Halman, J. (1991). Gender's role in responses to infertility. Psychology of Women Quarterly, 15, 295-316.

Bai, C., Cui, N., Xu, X., Mi, G., Sun, J., Shao, D., . . . Cao, F. (2019). Effectiveness of two guided self-administered interventions for psychological distress among women with infertility: a three-armed, randomized controlled trial. Human Reproduction, 34(7), 1235-1248. doi:10.1093/humrep/dez066

Belonoschkin, B. (1962). Psychosomatic factors and matrimonial infertility. International Journal of Fertility, 7, 29-36.

Berg, B., \& Wilson, J. (1991). Psychological functioning across stages of treatment for infertility. Journal of Behavioral Medicine, 1 1-26.

Blanchet, C., Babineau, V., Boivin, J., \& Ruchat, S. (2019). Cross-cultural Adaptation of the Cardiff Fertility Knowledge Scale and the Fertility Status Awareness Tool for the French-Canadian Population . Journal of Obstetrics and Gynaecology Canada, 41(2), 204-209. doi:https://doi.org/10.1016/j.jogc. 2018.04.002

Boivin, J. (2003). A review of psychosocial interventions in infertility. Social Science \& Medicine, 12, 2325-2341.

Boivin, J., \& Gameiro, S. (2015). Evolution of psychology and counseling in infertility. Fertility and Sterility, 104, 251-259.

Boivin, J., Appleton, T., Baetens, P., Baron, J., Bitzer, J., Corrigan, E., . . . Kentenich, H. (2001). Guidelines for counselling in infertility: outline version. Human Reproduction, 16(6), 13011304.

Boivin, J., Takefman, J., \& Braverman, A. (2011). The fertility quality of life (FertiQoL) tool: Development and general psychometric properties. Human Reproduction, 26, 20842091.

Borght, M., \& Wyns, C. (2018). Fertility and infertility: Definition and epidemiology. Clinical Biochemistry, 62, 2-10. doi:https://doi.org/10.1016/j.clinbiochem. 2018 .03 .012

Bunting, I., \& Boivin, J. (2010). Development and preliminary validation of the Fertility Status Awareness Toll: FertiSTAT. Human Reproduction, 25, 1722-1733.

Caplan, G. (1964). Principles of preventive psychiatry. New York: Basic Books.

CDC (2020, December 30). Centers for Disease Control and Prevention. Retrieved from National Center for Chronic Disease Prevention and Health Promotion, Division of Reproductive Health.

Chu, K., Zhang, Q., Han, H., Xu, C., Pang, W., Ma, Y., ... Li, W. (2017). A systematic review and meta-analysis of nonpharmacological adjuvant interventions for patients undergoing assisted reproductive technology treatment. International Journal of Gynecology \& Obstetrics, 3.

Cox, C. (1982). An interaction model of client healthbehavior: Theoretical prescription for nursing. Advances in nursing science, 5(1), 4156.

Creswel, J. (2017). Mindfulness interventions. Annual Review of Psychology, 491-516.

David, D. (2017). Tratat de Psihoterapii Cognitive și Comportamentale. Iași: Polirom.

De Berardis, D., Mazza, M., Del Nibletto, L., Serroni, N., Pino, M., Valchera, A., . . . Di Giannantonio, M. (2014). Psychopathology, emotional aspects and psychological counselling in infertility: a review. Clinical Therapeutics, 165(3), 163-169.

Deyhoul, N., Mohamaddoost, T., \& Hosseini, M. (2017). Infertility-Related Risk Factors: A Systematic Review. International Journal of Women's Health and Reproduction Sciences , 5(1), 24-29.

Domar, A., Seibel, M., \& Benson, H. (1990). The Mind/Body Program for Infertility: a new behavioral treatment approach for women with infertility. Fertility and Sterility, 53(2), 246249.

Donarelli, Z., Lo Coco, G., Gullo, S., Salerno, L.,

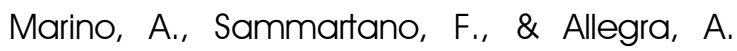
(2016). ORIGINAL ARTICLEPsychology and counsellingthe Fertility Quality of LifeQuestionnaire (FertiQoL) Relational subscale: psychometric properties and discriminant validity across gender. Human Reproduction, 2061-2071. doi::10.1093/humrep/dew168 
Doyle, M., \& Carballedo, A. (2014). Infertility and Mental Health. Advances in psychiatric treatment , 20, 297-303.

Eijkemans, M. (2014). Too old to have children? Lessons from natural fertility populations. Human Reproduction, 29(6), 1304-1312.

Eisendrath, S., Gillung, E., Delucchi, K., Segal, Z., Nelson, J., Mclnnes, L., . . Feldman, M. (2016). A randomized controlled trial of mindfulnessbased cognitive therapy for treatment-resistant depression. Psychotherapy and Psychosomatics, 2, 99-110.

Ergin, R., Polat, A., Kars, B., Öztekin, D., Sofuoğlu, K., \& Çalışkan, E. (2018). Social stigma and familial attitudes related to infertility. Turksih Journal of Obstetrics and Gynecology, 15(1), 46-49.

Erikson, E. (1964). Childhood and society (2nd ed.). New York: Norton.

ESHRE, (2002). Task Force on Ethics and Law. III. Gamete and embryo donation. Human Reproduction, 1407-1408.

Fischer, I. (1952). Psychogenic aspects of infertility. Fertility and Sterility, 4, 466-471.

Frederiksen, Y., Farver-Vestergaar, I., Skovgard, N., Ingerslev, H., \& Zachariae, R. (2015). Efficacy of psychosocial interventions for psychological and pregnancy outcomes in infertile women and men: a systematic review and metaanalysis. BMJ Open.

Galhardo, A., Cunha, M., \& Pinto-Gouveia, J. (2019). A 7-year follow-up study of the Mindfulness-Based Program for Infertility: Are there long-term effects. Clinical Psychology \& Psychotherapy, 26, 409-417.

Galst, J. (2017). The Elusive Connection Between Stress and Infertility: A Research Review With Clinical Implications. Journal of Psychotherapy Integration.

Gloster, A., Walder, N., Levin, M., Twohig, M., \& Karekla, M. (2020). The empirical status of acceptance and commitment therapy: A review of meta-analyses. Journal of Contextual Behavioral Science, 18, 181-192.

Greil, A., Schmidt, L., \& Peterson, B. (2014). Understanding and treating the psychosocial consequences of infertility. In A. Wenzel (Ed.), The Oxford Handbook of Perinatal Psychology. Oxford: Oxford University Press.

Hammerberg, K., Zosel, R., Comoy, C., Robertson, S., Holden, C., Deeks, M., \& Johnson, L. (2017). Fertility-related knowledge and informationseeking behaviour among people of reproductive age: a qualitative study. Human Fertility, 88-95.

Hanson, B., Johnstone, E., Dorais, J., Silver, B., Peterson, M., \& Hotaling, J. (2017). Female infertility, infertility-associated diagnoses, and comorbidities: a review. Journal of Assisted Reproduction and Genetics, 34(2), 167-177.

Hart, R. (2016). Physiological aspects of female infertility: role of the environment, modern lifestyle and genetics. Physiological Reviews, 96(3), 873-909.

Hasanzadeh, M., Akbari, B., \& Abolghasemi, A. (2019). Efficiency of Acceptance and Commitment Therapy on Psychological Wellbeing and Sexual Function in Women with Infertility History. Avicenna Journal of Nursing and Midwifery Care, 27(4), 250-259.

Hosseinpanahi, M., Mirghafourvand, M., FarshbafKhalili, A., Esmaeilpour, K., Rezaei, M., \& Malakouti, J. (2020). The effect of counseling based on acceptance and commitment therapy on mental health and quality of life among infertile couples: A randomized controlled trial. Journal of Education and Health Promotion, 9.

Inhorn, M., \& Patrizio, P. (2015). Infertility around the globe: new thinking on gender, reproductive technologies and global movements in the 21 st century. Human Reproduction Update, 21(4), $411-426$. doi:https://doi.org/10.1093/humupd/dmv016

Institute for Health Metrics and Evaluation, (2020, October 17). IHME. Retrieved from IHME: http://www.healthdata.org/romania?fbclid =lw AROHQHU9GGmh07FicaLNAykyXzvuK9bMd3Qf -U2XpraXpBGBySvVEoWI5Sc

Institute of Medicine, (2001). Crossing the Quality Chasm: A New Health System for the 21st Century. National Academy Press.

Jafarzadeh-Kenarsari, F., Ghahiri, A., ZarghamBoroujeni, A., \& Habibi, M. (2015). Exploration of the counseling needs of infertile couples: A qualitative study. Iranian Journal of Nursing and Midwifery Research, 20(5), 552-559. doi: 10.4103/1 735-9066.164506

Johnson, C., Greil, A., Shreffler, K., \& McQuillan, K. (2018). Fertility and Infertility: Toward an Integrative Research Agenda. Population Research and Policy Review, 37, 641-666.

Joy, J., \& McCrystal, P. (2015). The Role of Counselling in the Management of Patients with Infertility. The Obstetrician \& Gynaecologist, 17, 83-89. doi:10.1111/tog.12174

Karaca, A., Yazuvcan, A., Batmaz, S., Cangür, S., \& Çalişkan, A. (2019). The Effect of Cognitive Behavioral Group Therapy on Infertility Stress, General Health, and Negative Cognitions: A Randomized Controlled Trial. Journal of Rational-Emotive \& Cognitive-Behavior Therapy. doi:https://doi.org/10.1007/s10942019-00317-3

Kim, M., Kim, S., Chang, S., Yoo, J., Kim, H., \& Cho, J. (2014). Effect of a Mind-Body Therapeutic 
Program for Infertile Women Repeating In Vitro Fertilization Treatment on Uncertainty, Anxiety, and Implantation Rate. Asian Nursing Research, 8(1), 49-56.

Kırca, N., \& Pasinlioğlu, T. (2013). Psychosocial Problems During Infertility Treatment. Current Approaches in Psychiatry, 162-178.

Li, X., Wang, K., HuO, Y., \& Zhou, M. (2018). The effect of infertility-related stress on Chinese infertile females' mental health: The moderating role of marital adjustment. PsyCh Journal , 1-8.

Lipowski, Z. (1984). What does the word 'psychosomatic' really mean? A historical and semantic inquiry. Psychosomatic Medicine, 46, 153-171.

Luk, B., \& Loke, A. (2015). The Impact of Infertility on the Psychological Well-Being, Marital Relationships, Sexual Relationships, and Quality of Life of Couples: A Systematic Review. Journal of Sex \& Marital Therapy, 41(6), 610-625.

Maroufizadeh, S., Ghaheri, A., Almasi-Hashiani, A., Mohammadi, M., Navid, B., Ezabadi, Z., \& Samani, R. (2018). The prevalence of anxiety and depression among people with infertility referring to Royan Institute in Tehran, Iran: A cross-sectional questionnaire study. Middle East Fertility Society Journal, 23(2), 103-106.

Montgomery, K., Norman, P., Messenger, A., \& Thompson, A. (2016). The importance of mindfulness in psychosocial distress and quality of life in dermatology patients. British Journal of Dermatology, 5, 930-936.

Morone, N., Greco, C., Moore, C., Rollman, B., Lane, B., Morrow, L., . . . Weiner, D. (2016). A mind-body program for older adults with chronic low Back pain: a randomized clinical trial. JAMA Internal Medicine, 3, 329-337.

Moudi, Z., Piramie, R., Ghasemi, M., \& Ansari, H. (2019). Effect of an infertility counseling program on perceived stigma among infertile females candidates for intra-uterine insemination. Journal of Midwifery \& Reproductive Health, 7(4), 1870-1879.

Newton, C., Sherrard, W., \& Glavac, I. (1999). The fertility problem inventory: Measuring perceived infertility-related stress. Fertility and Sterility , 72, 54-62.

NICE, (2017, September 06). Fertility problems: assessment and treatment . Retrieved from National Institute for Health and Care Excellence:

https://www.nice.org.uk/guidance/cg156/chap ter/Recommendations\#principles-of-care

Ockhuijsen, H., van Smeden, M., van den Hoogen, A., \& Boivin, J. (2017). Validation study of the SCREENIVF: an instrument to screen women or men on risk for emotional maladjustment before the start of a fertility treatment. Fertility and Sterility, 107(6), 1370-1379. doi:https://doi.org/10.1016/j.fertnstert.2017.04. 008

Öztürk, B., Siyez, D., Esen, E., \& Kağnici, Y. (2020). Effects of infertility prevention programme on college students. Sexuality, Society and Learning, 20(5), 517-534.

Patel, A., Sharma, P., \& Kumar, P. (2018). Role of Mental Health Practitioner in Infertility Clinics: A Review on Past, Present and Future Directions. Journal of Human Reproductive Sciences, 11 (3), 219-228. doi:10.4103/jhrs.JHRS 4118

Patel, A., Sharma, P., Kumar, P. , \& Binu, $\bar{V}$. (2018). Sociocultural Determinants of Infertility Stress in Patients Undergoing Fertility Treatments. Journal of Human Reproductive Sciences, 172-179.

Pedro, J., Frederiksen, Y., Schmidt, L., Ingerslev, H., Zachariae, R., \& Martins, M. (2016). Comparison of three infertility-specific measures in men and women going through assisted reproductive technology treatment. Journal of Health Psychology, 1-12.

Peterson, B., Boivin, J., Norre, J., Smith, C., Thorn, P., \& Wischmann, T. (2012). An introduction to infertility counseling: a guide for mental health and medical professionals. Journal of Assisted Reproduction and Genetics, 29, 243-248.

Pinar, S., \& Yildirim, G. (2016). Psychological, Social and Ethical Dimensions of Infertility: A Review. International Journal of Nursing Didactis, 13-17.

Ravitsky, V., \& Kimmins, S. (2019). The forgotten men: rising rates of male infertility urgently require new approaches for its prevention, diagnosis and treatment. Biology of Reproduction, 872-874.

Rich, C., \& Domar, A. (2016). Addressing the emotional barriers to access to reproductive care. Fertility and Sterility, 105(5), 1124-1 127.

Ridenour, A., Yorgason, J., \& Peterson, B. (2009). The Infertility Resilience Model: Assessing Individual, Couple, and External Predictive Factors. Contemporary Family Therapy, 31, 3451.

Rouchou, B. (2013). Consequences of infertility in developing countries. Perspectives in Public Health, 174-179.

Sabarre, K., Khan, Z., Whitten, A., Remes, O., \& Phillips, K. (2013). A qualitative study of Ottawa university students' awareness, knowledge and perceptions of infertility, infertility risk factors and assisted reproductive technologies (ART). Reproductive Health, 10(41), 1-10.

Samadi, H., \& Doustkam, M. (2014). Investigating the effectiveness of Acceptance and Commitment Therapy (ACT) on marital compatibility and life expectancy in infertile 
women. International Academic Journal of Social Sciences, 1(1), 16-27.

Schweiger, U., Schweiger, J., \& Schweiger, J. (2018). Mental Disorders and Female Infertility. Archives of Psychology, 2(6), 1-14.

Sezgin, H., \& Hocaoğlu, C. (2014). Psychiatric aspects of infertility. Current Approaches in Psychiatry, 165-184.

Sobral, M., Costa, M., Schmidt, L., \& Martins, M. (2017). COMPI Fertility Problem Stress Scales is a brief, valid and reliable tool for assessing stress in patients seeking treatment. Human Reproduction, 32(2), 375-382. doi:https://doi.org/10. 1093/humrep/dew315

Springer, K., Levy, H., \& Tolin, D. (2018). Remission in CBT for adult anxiety disorders: A metaanalysis. Clinical psychology review, 61, 1-8. doi:https ://doi.org/10.1016/j.cpr.2018.03.002

Tola, E., Yalcin, S., Dugan, N., \& Oral, B. (2019). The association of type $D$ personality and depression with infertility in women. Journal of Psychosomatic Obstetrics \& Gynecology, 1-8.

Tsai, Y., Wang, T., Wei, H., Hsu, C., Ho, H., Chen, W., .... Chao, J. (2013). Dietary intake, glucose metabolism and sex hormones in womenwith polycystic ovary syndrome (PCOS) compared with women withnon-PCOS-related infertility. British Journal of Nutrition, 109, 2190-2198.

Verhaak, C., Lintsen, A., Evers, A., \& Braat, D. (2010). Who is at risk of emotional problems and how do you know? Screening of women going for IVF treatment. Human Reproduction, 25, 1234-1240.

WHO (2020, February 4). World Health Organization. Retrieved from WHO: https://www.who.int/news/item/04-02-2020multiple-definitions-of-infertility

Wiweko, B., Anggraheni, U., Elvira, S., \& Lubis, H. (2017). Distribution of stress level among infertility patients. Middle East Fertility Society Journal, 22(2), 145-148.
World Health Organization, (2001). International Classification of Functioning, Disability and Health. Geneva: WHO.

World Health Organization, (2002). International Statistical Classification of Diseases and Related Health Problems. Sydney: WHO.

Ying, L., Wu, L., \& Loke, A. (2015). Gender differences in experiences with and adjustments to infertility: A literature review. International Journal of Nursing Studies, 16401652.

Ying, L., Wu, L., \& Loke, A. (2016). he effects of psychosocial interventions onthe mental health, pregnancy rates, and marital function of infertilecouples undergoing in vitro fertilization: a systematic review. Journal of Assisted Reproduction and Genetics , 6, 689701.

Yusuf, L. (2016). Depression, anxiety and stress among female patients of infertility; A case control study. Pakistan Journal of Medical Sciences, 32(6), 1340-1343.

Zegers-Hochschild, F., Adamson, D., Dyer, S., Racowsky, C., de Mouzon, J., Sokol, R., . . . van der Poel, S. (2017). The International Glossary on Infertility and Fertility Care. Fertility and Sterility, 108(3), 393-406.

Zhou, R., Cao, Y., Liu, D., \& Xiao, J. (2021). Pregnancy or Psychological Outcomes of Psychotherapy Interventions for Infertility: A Meta-Analysis. Frontiers in Psychology, 12. doi: 10.3389/fpsyg.2021.643395

Zurlo, M., Cattaneo Della Volta, M., \& Vallone, F. (2017). Factor structure and psychometric properties of the Fertility Problem InventoryShort Form. Health Psychology Open, 1-11. doi: $10.1177 / 2055102917738657$

Zurlo, M., Cattateno Della Volta, M., \& Vallone, F. (2018). Predictors of quality of life and psychological health in infertile couples: the moderating role of duration of infertility. Quality of Life Research , 27, 945-954. 
MeCJ 2021 